\title{
Intérêt du scanner abdominopelvien chez les patients âgés de plus de 75 ans consultant aux urgences pour douleurs abdominales
}

\section{Usefulness of abdominal CT Scan for elderly patients with abdominal pain in an Emergency Department}

\author{
M. Lafay $\cdot$ C. Chenevier-Gobeaux $\cdot$ L. Paslaru $\cdot$ G. Lefèvre $\cdot$ M. Leveau $\cdot$ C. Gast $\cdot$ S. Houry $\cdot$ P. Ray \\ Reçu le 22 juin 2017; accepté le 20 janvier 2018 \\ (C) SFMU et Lavoisier SAS 2018
}

Résumé Introduction : Déterminer l'étiologie des syndromes abdominaux aigus (SAA) chez les personnes âgées nécessite souvent une imagerie abdominale. L'objectif de notre étude était d'étudier la place du scanner abdominal chez les patients âgés de plus de 75 ans consultant aux urgences, et de rechercher des facteurs prédictifs de scanner pathologique.

Patients et méthodes : Il s'agissait d'une étude rétrospective, monocentrique, observationnelle, effectuée aux

\footnotetext{
M. Lafay $\cdot$ M. Leveau $\cdot$ C. Gast $\cdot$ P. Ray $(\bowtie)$

Service d'accueil des urgences de l'hôpital Tenon, hôpitaux universitaires Est-Parisien,

Assistance publique-Hôpitaux de Paris (AP-HP),

4, rue de la Chine, F-75020 Paris, France

e-mail : patrick.ray@aphp.fr

C. Chenevier-Gobeaux

Service de diagnostic biologique automatisé, hôpital Cochin, hôpitaux universitaires Paris-Centre (HUPC) (AP-HP),

F-75014 Paris cedex, France

\section{Paslaru}

Service de radiologie de l'hôpital Tenon, hôpitaux universitaires Est-Parisien (AP-HP),

4, rue de la Chine, F-75020 Paris, France

G. Lefèvre

Biochimie et hormonologie de l'hôpital Tenon, hôpitaux universitaires Est-Parisien (AP-HP),

55, rue Pelleport, F-75020 Paris, France

\section{S. Houry}

Service de chirurgie viscérale de l'hôpital Tenon, hôpitaux universitaires Est-Parisien, Assistance publique-Hôpitaux de Paris (AP-HP),

4, rue de la Chine, F-75020 Paris, France

S. Houry · P. Ray

DHU Fight Aging and Stress (FAST),

Sorbonne université, Paris, F-75005, Paris, France
}

urgences de l'hôpital Tenon entre le 1er janvier et le 31 décembre 2014, chez les patients âgés de plus de 75 ans consultant pour un SAA. Deux groupes distincts étaient définis et comparés : un groupe « scanner normal » et un groupe « scanner pathologique », défini selon le compte-rendu du radiologue.

Résultats : Cent soixante-dix-huit patients (d'âge moyen de 84 ans) ont été inclus. Cent quatre-vingt-six scanners ont été effectués durant l'année 2014, parmi lesquels 41 scanners (22\%) étaient normaux. Les pathologies retrouvées le plus fréquemment étaient les occlusions (40\%), les pathologies urinaires $(23 \%)$, et les pathologies biliopancréatiques (22\%). Il n'existe aucun facteur clinique ou biologique prédictif de scanner pathologique.

Conclusion : Le scanner semble être un examen d'une grande rentabilité diagnostique chez la personne âgée, dont l'indication doit être large, car aucune donnée clinicobiologique n'est associée à un scanner pathologique.

Mots clés Scanner abdominal · Patients âgés · Douleur abdominale

\footnotetext{
Abstract Introduction: Most of the time, abdominal imaging is necessary for abdominal pain in elderly patients. The aim of this study was to evaluate the usefulness of computerized tomography (CT) scan for elderly patients in the emergency department, and seek for predictive factors of pathologic CT scan.

Procedure: This retrospective monocentric study was carried out at the Tenon's hospital emergency department in Paris from January 1, 2014, to December 31, 2014, and included patients aged over 75 years with abdominal pain. Two groups were established: one group "normal CT scan" and the other group "pathologic CT scan", defined by the radiologist summary.

Results: The average age of 178 patients was 84 years old; 186 CT scans were performed in 2014, among which only 41
} 
CT scans were normal (22\%). Most frequent pathologies diagnosed were bowel obstructions (40\%), urinary tractus diseases $(23 \%)$ and biliary tractus diseases $(22 \%)$. Our analysis showed that there is not clinical or biological variable predictive of pathologic CT scan.

Conclusion: CT scan seems to be a useful exam for investigating abdominal pain in elderly patients.

Keywords Abdominal CT scan · Elderly patients · Abdominal pain

\section{Introduction}

Les personnes de plus de 75 ans représentent $12 \%$ des passages aux urgences, parmi lesquelles $10 \%$ se présentent pour un motif de gastroentérologie et $6 \%$ ressortent avec comme diagnostic principal une pathologie digestive [1]. Les patients âgés présentent parfois une clinique atypique, peu spécifique, pouvant faire évoluer leur pathologie jusqu'à un haut niveau de gravité avant de s'exprimer par des symptômes francs [2]. Les étiologies des syndromes abdominaux aigus (SAA) chez les personnes âgées sont donc parfois difficiles à déterminer, et nécessitent le plus souvent une imagerie abdominale. L'abdomen sans préparation (ASP) n'est plus recommandé dans les douleurs abdominales aiguës du fait d'une sensibilité et d'une spécificité très faibles [3,4]. L'échographie abdominale reste recommandée en première intention pour la recherche des pathologies hépatobiliaires, notamment une cholécystite. Le scanner abdominal est recommandé en première intention pour la recherche de diverticulite, et pour la recherche d'autres pathologies chirurgicales (occlusion...) $[5,6]$. Les recommandations ainsi que la littérature disponible suggèrent que le scanner serait l'imagerie à privilégier dans la majorité des cas pour le bilan étiologique d'un SAA chez le sujet âgé [7].

L'objectif de notre étude était d'étudier la place du scanner abdominal chez les patients âgés de plus de 75 ans consultant aux urgences et de déterminer s'il existait des facteurs prédictifs de scanner pathologique.

\section{Patients et méthodes}

Il s'agissait d'une étude rétrospective, monocentrique, observationnelle, effectuée au sein du service d'accueil des urgences de l'hôpital Tenon entre le $1^{\text {er }}$ janvier et le 31 décembre 2014. En 2014, 46181 patients ont consulté au service d'accueil des urgences (SAU) de l'hôpital Tenon (soit environ 125 patients par jour) dont 6289 (14\%) personnes âgées de plus de 75 ans. Une unité d'hospitalisation $(\mathrm{UH})$ de 14 lits (service « portes ») est associée au SAU.

Cette étude rétrospective ne modifiait pas la prise en charge des patients, et était donc hors loi Huriet. Ainsi, le consentement des patients (dont les résultats ont été évidemment anonymisés) n'a pas été nécessaire. Était inclus tout patient de plus de 75 ans lors de sa consultation ayant bénéficié durant sa prise en charge aux urgences ou à l'UH d'un scanner abdominopelvien, pour un motif principal digestif. Étaient exclus ceux présentant une douleur d'origine post-traumatique, ceux pour lesquels l'indication du scanner n'était pas en rapport avec un SAA au premier plan (débrouillage...), ceux pour lesquels le scanner avait été effectué après le passage au SAU (dans un service d'aval). Nous avons récupéré la liste des patients de plus de 75 ans ayant bénéficié d'un scanner durant l'année 2014 auprès du service d'imagerie, puis les données cliniques du dossier médical des urgences. Les données recueillies dans les comptes-rendus de passage aux urgences (CRU) étaient les caractéristiques clinicobiologiques et démographiques usuelles, les antécédents principaux notables, la prise éventuelle d'antibiotiques dans les sept derniers jours et les antécédents de chirurgie abdominale. Les anomalies de l'examen clinique ont été notées (douleur abdominale, défense...), de même que les résultats d'examens biologiques de routine. La suspicion diagnostique préscanner, lorsqu'elle était précisée dans le dossier, a été notée ainsi que le résultat du scanner. Les thérapeutiques (dont les antalgiques, les antibiotiques, ou l'aspiration digestive) ainsi que le devenir et le décès hospitalier ont été recueillis. Nous nous sommes aidés du dossier médical (UrQual ${ }^{\mathbb{Q}}$, Maincare Solutions, Cestat, France), du compte-rendu opératoire et du compte-rendu d'hospitalisation pour les autres données recueillies. Nous avons considéré le compte-rendu du scanner dicté par le radiologue qui l'a interprété au moment de l'examen les images. Souvent, il s'agissait du compte-rendu du senior, mais parfois lu uniquement par un interne de garde, mais les scanners sont ensuite relus dans les 24 heures par un sénior et donc nous pouvons considérer que tous les scanners ont été seniorisés. Nous avons séparé les scanners en normal ou pathologique (quelle que soit la pathologie retrouvée : urgence chirurgicale vraie type perforation digestive, sigmoïdite, fécalome, colique néphrétique, etc.) et nous avons classé les pathologies par grands syndromes (occlusion, pathologie inflammatoire, pathologie hépatobiliaire et pathologie vasculaire).

\section{Analyse statistique}

Les analyses statistiques ont été effectuées sur les logiciels Microsoft Office Excel ${ }^{\circledR} 2016$ (Microsoft Corporation, Redmond, États-Unis) et MedCalc ${ }^{\circledR}$ (MedCalc Software, Mariarkerke, Belgique). Les données étaient présentées 
en moyenne et écart-type pour les variables quantitatives et valeur et pourcentage pour les valeurs qualitatives. Les analyses comparatives ont été réalisées en situation bilatérale selon le test $\mathrm{du} \mathrm{Chi}^{2}$ de Pearson pour les comparaisons des variables qualitatives et selon le test de Student pour les comparaisons des variables quantitatives. Les caractéristiques cliniques et biologiques des patients ont été comparées entre le groupe « scanner normal » et le groupe " scanner pathologique ». Les résultats étaient analysés à l'aide du logiciel Med Calc 3.4.2.0 pour Windows. Tous les tests étaient bilatéraux. Une valeur de $p$ inférieure à 0,05 était considérée comme significative.

\section{Résultats}

Au total, 186 scanners ont été effectués durant l'année 2014 pour 178 patients (Fig. 1). En effet, huit patients ont bénéficié de deux scanners au cours de l'année 2014 (trois hommes, cinq femmes). Cent neuf scanners étaient effectués sans, puis avec injection. Les principales données cliniques des patients sont résumées dans le tableau 1. Soixante-dix (38\%) patients vivaient à domicile. Le résultat de la bandelette urinaire (BU) était consigné dans 26 dossiers (14\%) : huit étaient négatives, dix-huit positives. Tous les patients ont bénéficié d'un bilan biologique (comprenant +/- la CRP, Bilan hépatique, procalcitonine...) durant leur prise en charge au SAU, dont trois n'étaient pas disponibles pour notre étude. Néanmoins, aucune variable clinique ou biologique n'orientait vers un scanner anormal (Tableaux 1 et 2). Lorsqu'une douleur était retrouvée à la palpation abdominale, celle-ci était le plus souvent diffuse (23 patients) ou localisée en fosse iliaque gauche (18 patients) et dans les fosses lombaires (17 patients). Cinquante-cinq patients avaient une palpation abdominale normale (abdomen souple à l'examen clinique), $11 \%$ d'entre eux avaient une défense à l'examen clinique et aucun ne présentait de contracture abdominale. L'évaluation de la douleur n'était consignée que dans 50 dossiers ( $27 \%$ des dossiers). Parmi ces dossiers, l'échelle de mesure était l'échelle numérique dans 49 dossiers, l'Algoplus dans un dossier. Dans deux dossiers, la douleur était cotée "Non évaluable ». Dix-sept patients avaient une échelle visuelle analogique (EVA) à $0 / 10$. Un patient était coté à 10/10 et l'EVA était supérieure à 7/10 dans six dossiers. Au total 129 patients (69\%) ont bénéficié d'une prescription d'antalgiques durant leur prise en charge aux urgences, dont $9 \%$ ont reçu des antalgiques de palier 3 . Le tableau 2 montre les résultats retrouvés au scanner établis

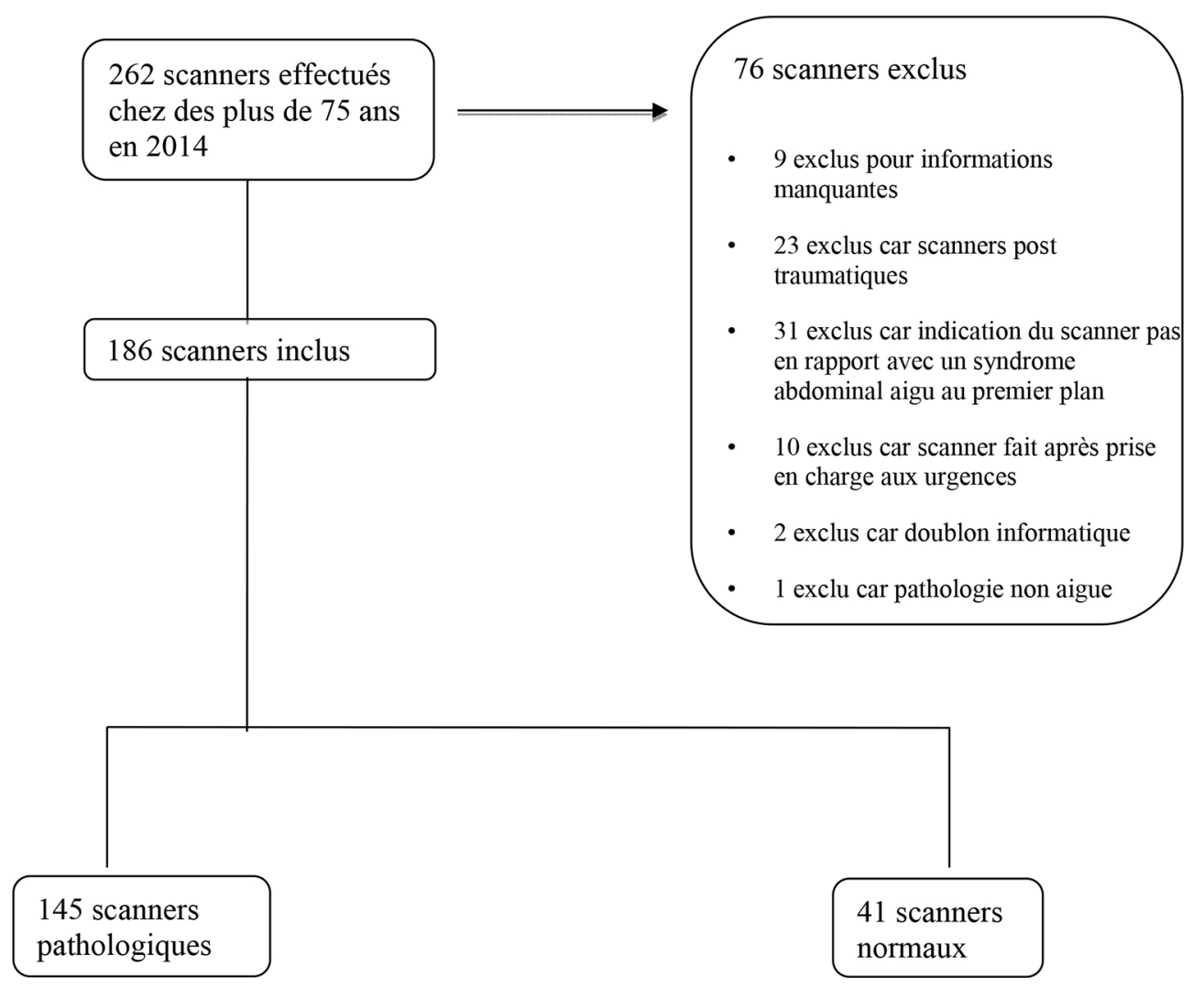

Fig. 1 Diagramme de flux 
Tableau 1 Caractéristiques cliniques et biologiques des patients

\begin{tabular}{|c|c|c|c|}
\hline & $\begin{array}{l}\text { Scanner pathologique } \\
N=145\end{array}$ & $\begin{array}{l}\text { Scanner normal } \\
N=41\end{array}$ & Valeur de $p$ \\
\hline Âge (années) & $84 \pm 6$ & $83 \pm 5$ & 0,30 \\
\hline Hommes & $63(43 \%)$ & $18(44 \%)$ & 0,90 \\
\hline Femmes & $82(57 \%)$ & $23(56 \%)$ & 0,90 \\
\hline Hypertension artérielle & $88(60 \%)$ & $30(73 \%)$ & 0,20 \\
\hline BPCO & $18(12 \%)$ & $5(12 \%)$ & 0,81 \\
\hline Insuffisance cardiaque & $49(33 \%)$ & $18(44 \%)$ & 0,33 \\
\hline Troubles cognitifs & $32(22 \%)$ & $15(39 \%)$ & 0,12 \\
\hline Séquelle d'AVC & $27(18 \%)$ & $7(17 \%)$ & 0,99 \\
\hline Diabète & $28(19 \%)$ & $14(34 \%)$ & 0,07 \\
\hline Antécédent de chirurgie abdominale & $69(47 \%)$ & $17(41 \%)$ & 0,60 \\
\hline Antibiothérapie récente & $5(3 \%)$ & $4(9 \%)$ & 0,21 \\
\hline Fréquence cardiaque (bpm) & $85 \pm 20$ & $81 \pm 16$ & 0,29 \\
\hline PAm (mmHg) & $94 \pm 20$ & $94 \pm 20$ & 0,93 \\
\hline Température moyenne $\left({ }^{\circ} \mathrm{C}\right)$ & $37 \pm 0,9$ & $37 \pm 0,9$ & 0,29 \\
\hline $\mathrm{SpO}_{2}(\%)$ & $95 \pm 3$ & $95 \pm 3$ & 0,67 \\
\hline Marbrures & $7(\%)$ & $2(\%)$ & 0,69 \\
\hline Défense abdominale & $15(\%)$ & $5(\%)$ & 0,93 \\
\hline Leucocytes $\left(10^{3} / \mathrm{ml}\right)$ & $\begin{array}{l}12877 \pm 7243 \\
(n=142)\end{array}$ & $\begin{array}{l}11821 \pm 799 \\
(n=41)\end{array}$ & 0,38 \\
\hline Neutrophiles $\left(10^{3} / \mathrm{ml}\right)$ & $\begin{array}{l}10736 \pm 585 \\
(n=142)\end{array}$ & $\begin{array}{l}9251 \pm 836 \\
(n=41)\end{array}$ & 0,20 \\
\hline CRP (mg/l) & $\begin{array}{l}87 \pm 97 \\
(n=23)\end{array}$ & $\begin{array}{l}83 \pm 93 \\
(n=72)\end{array}$ & 0,87 \\
\hline Lactate $(\mathrm{mmol} / \mathrm{l})$ & $\begin{array}{l}2,5 \pm 1,5 \\
(n=7)\end{array}$ & $\begin{array}{l}3,3 \pm 2,0 \\
(n=12)\end{array}$ & 0,32 \\
\hline Natrémie (mmol/l) & $\begin{array}{l}137 \pm 5,2 \\
(n=140)\end{array}$ & $\begin{array}{l}137 \pm 5,8 \\
(n=41)\end{array}$ & 0,56 \\
\hline Créatinine $(\mu \mathrm{mol} / \mathrm{l})$ & $\begin{array}{l}116 \pm 76 \\
(n=138)\end{array}$ & $\begin{array}{l}151 \pm 122 \\
(n=41)\end{array}$ & 0,025 \\
\hline ASAT (UI/l) & $\begin{array}{l}82 \pm 108 \\
(n=95)\end{array}$ & $\begin{array}{l}36 \pm 18 \\
(n=22)\end{array}$ & 0,05 \\
\hline ALAT (UI/1) & $\begin{array}{l}63 \pm 107 \\
(n=95)\end{array}$ & $\begin{array}{l}30 \pm 30 \\
(n=22)\end{array}$ & 0,16 \\
\hline Bilirubine totale $(\mu \mathrm{mol} / \mathrm{l})$ & $\begin{array}{l}31 \pm 62 \\
(n=94)\end{array}$ & $\begin{array}{l}20 \pm 37 \\
(n=22)\end{array}$ & 0,44 \\
\hline Lipase (UI/l) & $\begin{array}{l}410 \pm 1796 \\
(n=38)\end{array}$ & $\begin{array}{l}32 \pm 28 \\
(n=14)\end{array}$ & 0,43 \\
\hline
\end{tabular}

par le radiologue, parmi lesquels 41 (22\%) scanners sont considérés comme normaux. Au total, 135 patients $(73 \%)$ ont été hospitalisés (dont 22 perdus de vue). Parmi les 113 patients dont le devenir est connu, neuf patients ont nécessité d'être pris en charge au bloc opératoire en urgence
(5\%) et $21(11 \%)$ sont décédés durant leur prise en charge aux urgences ou en hospitalisation. Six patients (3\%) sont décédés aux urgences. Quarante-cinq patients (24\%) sont rentrés au domicile au décours de leur prise en charge aux urgences. 


\begin{tabular}{|c|c|}
\hline & $\begin{array}{l}\text { Scanner } \\
\text { pathologique } \\
N=145\end{array}$ \\
\hline Occlusion & $58(40 \%)$ \\
\hline Intraluminale : & 20 \\
\hline Fécalome/Stase stercorale & 1 \\
\hline Corps étranger & 10 \\
\hline Extraluminale & 5 \\
\hline Bride & 9 \\
\hline Volvulus & 8 \\
\hline Hernie & 3 \\
\hline Carcinose & 2 \\
\hline \multicolumn{2}{|l|}{ Perforation } \\
\hline \multicolumn{2}{|l|}{ Fonctionnelle } \\
\hline Pathologies inflammatoires & $12(8 \%)$ \\
\hline Diverticulite & 4 \\
\hline Colite infectieuse & 4 \\
\hline Collection/abcès/fistule & 3 \\
\hline Fasciite nécrosante de la marge anale & 1 \\
\hline Pathologies pancréatobiliaires & $32(22 \%)$ \\
\hline Cholécystite & 13 \\
\hline Angiocholite & 5 \\
\hline Dilatation des voies biliaires & 6 \\
\hline Pancréatite & 2 \\
\hline Masse pancréas & 6 \\
\hline Pathologies urinaires & $33(23 \%)$ \\
\hline Pyélonéphrite & 4 \\
\hline Lithiase urinaire non compliquée & 2 \\
\hline Lithiase urinaire compliquée & 23 \\
\hline Globe urinaire & 1 \\
\hline Kyste rénal & 1 \\
\hline Atrophie de greffon rénal & 1 \\
\hline Masse vésicale & 1 \\
\hline Pathologies vasculaires & $8(5 \%)$ \\
\hline Ischémie mésentérique & 5 \\
\hline Hématome & 5 \\
\hline
\end{tabular}

\section{Discussion}

Notre travail montre que le scanner est anormal dans près de $80 \%$ des cas chez les personnes âgées. De plus, il n'existe pas de critères « simples » (cliniques ou biologiques) qui permettraient au clinicien (urgentiste ou chirurgien) de prédire une anomalie au scanner abdominopelvien.

Les données recueillies soulignent la difficulté de l'examen clinique chez la personne âgée : si de nombreux patients avaient une infection intra-abdominopelvienne, très peu présentaient des signes cliniques de sepsis (fièvre dans seulement $18 \%$ ), et peu étaient hypotendus ou présentaient des marbrures. De même, si la majorité des patients avaient un scanner pathologique, seuls $11 \%$ d'entre eux avaient une défense à l'examen clinique et aucun ne présentait de contracture.

Notre travail est comparable aux autres études (Tableau 3) : les diagnostics les plus fréquemment retrouvés sont dans la majorité des études les occlusions, les pathologies urinaires et les pathologies biliopancréatiques. Le nombre de scanners pathologiques est en revanche très variable d'une étude à l'autre. Plusieurs choses peuvent expliquer cela : la difficulté de définir ce qu'est un scanner normal, les variabilités du recours au scanner d'un centre à l'autre et également les différences de prise en charge. Toutes ces études posent la question de l'intérêt du scanner abdominal en termes de prise en charge thérapeutique chez les personnes âgées (changement de thérapeutique, hospitalisation, coût final ?), ainsi que de l'intérêt d'un scanner systématique en cas de SAA chez une personne âgée. Garner et al [8] ont étudié de 2006 à 2013, 464 scanners de patients de plus de 80 ans s'étant présentés aux urgences pour SAA. La tomodensitométrie influençait de façon majeure la prise en charge, avec une modification de traitement dans $65 \%$ des cas, une prise en charge chirurgicale dans $48 \%$. L'étude de Millet et al [13] a montré que lorsqu'un scanner abdominal était effectué de façon systématique chez tous les patients âgés se présentant pour SAA, celui-ci permettait de diagnostiquer $30 \%$ de pathologies intra-abdominales de plus. Enfin, l'étude de Barksdale et al. [14], qui ne s'intéressait pas spécifiquement aux sujets âgés, incluait 547 scanners en 2011, a montré que $54 \%$ des diagnostics cliniques initiaux étaient modifiés par le résultat du scanner, et que la volonté d'hospitaliser les patients diminuait de $20 \%$ après le scanner.

Dans notre travail, aucun marqueur biologique n'était significativement différent entre la population des patients ayant un scanner normal et ceux ayant un scanner pathologique. La faible proportion de patients ayant bénéficié d'un dosage de CRP dans notre service n'a pas permis d'évaluer la pertinence de ce biomarqueur, mais une méta-analyse suggérait un intérêt modeste dans les pathologies intraabdominales [15].

Il s'agit d'une étude rétrospective et monocentrique, où les dossiers ont été récupérés et analysés a posteriori, avec des données recueillies à partir du dossier médical, ce qui favorise une non-exhaustivité (absence du devenir hospitalier pour 22/135 patients). Il aurait été intéressant de pouvoir comparer ces résultats à ceux d'autres services d'urgence où l'organisation des soins est différente, avec un accès à l'imagerie (notamment de garde) et un accès à un avis spécialisé différents. Notre étude n'a pas évalué le devenir des patients âgés s'étant présentés aux urgences pour SAA, sortis à domicile et n'ayant pas eu de scanner (taux de réadmission, ou de diagnostics manqués potentiellement). Nous n'avons pas non plus évalué l'évolution de la créatinine après injection 


\begin{tabular}{|c|c|c|c|c|}
\hline Étude & $\begin{array}{l}\text { Âge } \\
\text { des patients }\end{array}$ & $\begin{array}{l}\text { Nombre } \\
\text { de patients }\end{array}$ & $\begin{array}{l}\text { Pourcentage } \\
\text { de scanners } \\
\text { normaux }\end{array}$ & Pathologies les plus fréquemment retrouvées \\
\hline Gardner et al, 2013 [8] & $>80$ ans & 464 & $45 \%$ & $\begin{array}{l}\text { Occlusions } \\
\text { Diverticulites }\end{array}$ \\
\hline Cuccioli et al, 2013 [9] & $>75$ ans & 337 & $30 \%$ & $\begin{array}{l}\text { Occlusions } \\
\text { Pathologies inflammatoires } \\
\text { Pathologies urinaires }\end{array}$ \\
\hline Esses et al, $2001[10]$ & $>65$ ans & 104 & $37 \%$ & $\begin{array}{l}\text { Diverticulites } \\
\text { Cholécystites }\end{array}$ \\
\hline Perreard 2013 [11] & $>70$ ans & 100 & $8 \%$ & $\begin{array}{l}\text { Occlusions } \\
\text { Pathologies vasculaires } \\
\text { Diverticulites }\end{array}$ \\
\hline Hustey et al, 2005 [12] & $>75$ ans & 337 & $43 \%$ & $\begin{array}{l}\text { Occlusions } \\
\text { Diverticulites } \\
\text { Lithiases urinaires }\end{array}$ \\
\hline Millet et al, 2016 [13] & $>75$ ans & 401 & $11 \%$ & $\begin{array}{l}\text { Fécalomes } \\
\text { Occlusions } \\
\text { Pathologies biliaires } \\
\text { Pyélonéphrites }\end{array}$ \\
\hline Barksdale et al, 2011 [14] & Tout âge & 547 & $50 \%$ & $\begin{array}{l}\text { Lithiases urinaires } \\
\text { Appendicite } \\
\text { Diverticulite }\end{array}$ \\
\hline Notre étude & $>75$ ans & 186 & $22 \%$ & $\begin{array}{l}\text { Occlusions } \\
\text { Pathologies pancréatobiliaires } \\
\text { Pathologies urinaires }\end{array}$ \\
\hline
\end{tabular}

de produit de contraste chez ces patients fragiles. Enfin, nous avons recueilli le compte-rendu radiologique (interne ou senior) au moment de l'examen, et n'avons pas fait relire de façon systématique et en insu tous les examens par un ou plusieurs radiologues spécialisés. Néanmoins, au moment de la relecture des CRH, nous n'avons pas retrouvé de discordance suffisamment importante pour qu'un patient passe d'un groupe à l'autre. Il aurait également été intéressant d'étudier l'intérêt sur la prise en charge pré et post-scanner par l'urgentiste (changement de thérapeutique, hospitalisations, retour à domicile) [13]. Enfin, il aurait été intéressant d'évaluer l'impact économique : le scanner peut initialement représenter un surcoût, mais permet peut-être une réduction globale des dépenses en réduisant le nombre d'hospitalisations inutiles.

\section{Conclusion}

Le scanner abdominopelvien semble être un examen d'une grande rentabilité diagnostique chez la personne âgée, dont l'indication devrait être large. Cette indication est d'autant plus valable qu'aucun critère clinique ou biologique ne peut être retenu comme prédictif de scanner pathologique.

Conflit d'intérêts : les auteurs déclarent ne pas avoir de liens d'intérêts.

\section{Références}

1. Direction de la Recherche, des Études, de l'Évaluation et des Statistiques (DREES) (2014). Colloque DREES 18/11/2014 : Résultat du questionnaire patient - le parcours des personnes âgées aux urgences. http://drees.social-sante.gouv.fr/IMG/pdf/session_3_resultats_pa.pdf (Dernier accès le 6 février 2017)

2. Fagbohun CF, Toy EC, Baker B (1999) The evaluation of acute abdominal pain in the elderly patient. In: Primary Care Update for OB/GYNS 6. Elsevier Masson Ed, Paris, pp 181-185

3. Bugliosi TF, Meloy TD, Vukov LF (1990) Acute abdominal pain in the elderly. Ann Emerg Med 19:1383-6

4. Haute Autorité de santé (2009) Principales indications et «nonindications » de la radiographie de l'abdomen sans préparation. http:/www.has-sante.fr/portail/upload/docs/application/pdf/200902/texte_court_rx_asp.pdf (Dernier accès le 6 février 2017)

5. Société française de radiologie (2013) Guide du bon usage des examens d'imagerie médicale. http://gbu.radiologie.fr (Dernier accès le 6 février 2017) 
6. Van Randen V, Laméris A, Luitse W, et al (2011) The role of plain radiographs in patients with acute abdominal pain at the ED. Am J Emerg Med 29:582-9.e2

7. Laméris W, Randen A, Van ES SW, et al (2009) Imaging strategies for detection of urgent conditions in patients with acute abdominal pain: diagnostic accuracy study. BMJ 338:b2431

8. Gardner CS, Jaffe TA, Nelson RC (2015) Impact of CT in elderly patients presenting to the emergency department with acute abdominal pain. Abdom Imaging 40:2877-82

9. F. Cuccioli et al (2013). Société Française de Radiologie : Intérêt du scanner dans le bilan des douleurs abdominales aigues non traumatiques chez les patients âgés de plus de 75 ans. http://pe. sfrnet.org/Data/ModuleConsultationPoster/pdf/2013/1/9ad6706cff4f-48e4-b595-40440e81d11d.pdf (Dernier accès le 6 février 2017)

10. Esses D, Birnbaum A, Bijur P, et al (2004) Ability of CT to alter decision making in elderly patients with acute abdominal pain. Am J Emerg Med 22:270-2

11. Perreard A (2013) Performance diagnostique du scanner abdominopelvien sans injection pour les douleurs abdominales aiguës chez la personne âgée. https://www.google.fr/url?sa=t\&rct=j\&q=\&esrc=
s\&source=web\&cd=1\&ved=0ahUKEwienpzSqfvRAhWC7BQK Ha0_DvgQFggaMAA\&url=http\%3A\%2F\%2Farchive.bu.univnantes. fr $\% 2$ Fpollux $\% 2$ Ffichiers\%2Fdownload\%2Ff4e64793-b0ef48ca-ae32-d4c9a41280a1\&usg=AFQjCNGGk3unlu_f0f5ay8qI0IfmSzhhA\&sig2=VjtPRYgGX00AoEl4uM-

uOA\&bvm=bv.146094739,d.d24 (Dernier accès le 6 février 2017)

12. Hustey FM, Meldon SW, Banet GA, et al (2005) The use of abdominal computed tomography in older ED patients with acute abdominal pain. Am J Emerg Med 23:259-65

13. Millet I, Sebbane M, Molinari N, et al (2017) Systematic unenhanced CT for acute abdominal symptoms in the elderly patients improves both emergency department diagnosis and prompt clinical management. Eur Radiol 27:868-77

14. Barksdale AN, Hackman, JL, Gaddis, M, Gratton MC (2015) Diagnosis and disposition are changed when board-certified emergency physicians use CT for non-traumatic abdominal pain. Am J Emerg Med 33:1646-50

15. Gans SL, Atema, JJ, Stoker J, et al (2015) C-Reactive Protein and white blood cell count as triage test between urgent and nonurgent conditions in 2961 patients with acute abdominal pain. Medicine 94:e569 